\title{
On the admissible families of components of Hamming codes
}

\author{
Alexander M. Romanov \\ Sobolev Institute of Mathematics \\ 630090 Novosibirsk, Russia \\ rom@math.nsc.ru
}

\begin{abstract}
In this paper, we describe the properties of the $i$-components of Hamming codes. We suggest constructions of the admissible families of components of Hamming codes. It is shown that every $q$-ary code of length $m$ and minimum distance 5 (for $q=3$ the minimum distance is 3 ) can be embedded in a $q$-ary 1-perfect code of length $n=\left(q^{m}-1\right) /(q-1)$. It is also shown that every binary code of length $m+k$ and minimum distance $3 k+3$ can be embedded in a binary 1-perfect code of length $n=2^{m}-1$.
\end{abstract}

Keywords: Hamming codes, 1-perfect codes, q-ary codes, binary codes, i-component.

\section{Introduction}

Let $\mathbb{F}_{q}^{n}$ be a vector space of dimension $n$ over the Galois field $\mathbb{F}_{q}$. The Hamming distance between two vectors $\mathbf{x}, \mathbf{y} \in \mathbb{F}_{q}^{n}$ is the number of coordinates in which they differ and it is denote by $d(\mathbf{x}, \mathbf{y})$. An arbitrary subset $C$ of $\mathbb{F}_{q}^{n}$ is called $q$-ary 1-perfect code of length $n$, if for every vector $\mathbf{x} \in \mathbb{F}_{q}^{n}$ there exists a unique vector $\mathbf{c} \in C$ such that $d(\mathbf{x}, \mathbf{c}) \leq 1$. It is known that $q$-ary 1-perfect codes of length $n$ exist only if $n=\left(q^{m}-1\right) /(q-1)$, where $m$ is a natural number not less than two. We shall assume that the all-zero vector $\overrightarrow{0}$ is in code. A code is called linear if it is a linear space over $\mathbb{F}_{q}$. The linear 1-perfect codes are called Hamming codes. The $q$-ary Hamming code of length $n$ is denoted by $\mathbb{H}$.

The weight of a vector $\vec{x} \in \mathbb{F}_{q}^{n}$ is the number $d(\vec{x}, \overrightarrow{0})$. A vector of weight 3 of the code $\mathbb{H}$ is called triple. Consider the subspace $R_{i}$ spanned by the set of all triples of the code $\mathbb{H}$ having 1 in the $i$-th coordinate. All cosets $R_{i}+\vec{u}$ form the set of $i$-components of the $q$-ary Hamming code $\mathbb{H}$, where $i \in\{1,2 \ldots n\}, \vec{u} \in \mathbb{H}$. A family of components 
$R_{i_{1}}+\vec{u}_{1}, R_{i_{2}}+\vec{u}_{2}, \ldots, R_{i_{t}}+\vec{u}_{t}$ of the $q$-ary Hamming code $\mathbb{H}$ is called admissible if for $r, s \in\{1,2, \ldots, t\}, r \neq s$, we have $\left(R_{i_{r}}+\vec{u}_{r}\right) \cap\left(R_{i_{s}}+\vec{u}_{s}\right)=\varnothing$. See [5].

Let $n_{1} \leq n_{2}, C_{1} \subseteq \mathbb{F}_{q}^{n_{1}} \quad C_{2} \subseteq \mathbb{F}_{q}^{n_{2}}$. We lengthen all the vectors of the code $C_{1}$ to the length of $n_{2}$ by appending a zero vector of length $n_{2}-n_{1}$. They say that the code $C_{1}$ can be embedded in the code $C_{2}$ if all the lengthened vectors of $C_{1}$ belong to $C_{2}$. We consider all the vectors of the code $C_{2}$ in which the last $n_{2}-n_{1}$ coordinates are equal to zero. We delete the last $n_{2}-n_{1}$ coordinate in all such vectors. If the resulting set of shortened vectors coincides with $C_{1}$, then we say that the code $C_{1}$ can be embedded in the code $C_{2}$ in the strong sense.

Avgustinovich and Krotov [2] showed that any binary code of length $m$ and minimum distance 3 can be embedded (in the strong sense) in a binary 1-perfect code of length $2^{m}-1$.

In this paper, we describe properties of the $i$-components of Hamming codes. We suggest constructions of the admissible families of components of Hamming codes. It is shown that every $q$-ary code of length $m$ and minimum distance 5 (for $q=3$ the minimum distance is 3) can be embedded in a $q$-ary 1-perfect code of length $n=\left(q^{m}-1\right) /(q-1)$. It is also shown that every binary code of length $m+k$ and distance $3 k+3$ can be embedded in a binary 1-perfect code of length $n=2^{m}-1$.

We present three examples of the admissible families of components of Hamming codes. In Example 1, for an arbitrary $q$-ary code $(\Lambda \cup\{\overrightarrow{0}\}) \subset \mathbb{F}_{q}^{m}$ with minimum distance 5 we construct an admissible family of components of $q$-ary Hamming code of length $n=\left(q^{m}-1\right) /(q-1)$. The admissible family of component is constructed so that switching the components of this family, we obtain a $q$-ary 1 -perfect code $\mathbb{T}$ of length $n$ in which can be embedded the $q$-ary code $\Lambda \cup\{\overrightarrow{0}\}$ of length $m$. In Example 2, for an arbitrary ternary code of length $m$ and distance 3 in exactly the same method as in Example 1 we constructing an admissible family of components of the ternary Hamming code of length $n=\left(3^{m}-1\right) / 2$. In Example 3, for an arbitrary binary code of length $m+k$ and distance $3 k+3$ we constructing an admissible family of component of the binary Hamming code of length $n=2^{m}-1$. The admissible families of components from Examples 2 and 3 have the same properties as the admissible family of components from Example 1 and allow us, by switching the components, to construct the 1-perfect codes in which can be embedded codes of smaller length.

In Section 2 we present theorems describing the properties of the $i$-components of code $\mathbb{H}$. In Section 3 we describe the constructions of admissible families of components of code $\mathbb{H}$. In Section 4 we give Examples 2 and 3. In Section 5 we prove a theorem on the embeddability.

The parity-check matrix $H$ of the code $\mathbb{H}$ of length $n=\left(q^{m}-1\right) /(q-1)$ consists of $n$ pairwise linearly independent column vectors $\vec{h}_{i}$. The transposed column vector $\vec{h}_{i}$ belongs to $\mathbb{F}_{q}^{m}, i \in\{1, \ldots, n\}$. We assume that the columns of the parity-check matrix $H$ 
are arranged in some fixed order. The set $\mathbb{F}_{q}^{m} \backslash\{\overrightarrow{0}\}$ generates a projective space $P G_{m-1}(q)$ of dimension $(m-1)$ over the Galois field $\mathbb{F}_{q}$. In this space, points correspond to the columns of the parity-check matrix $H$ and the three points $i, j, k$ lie on the same line if

the corresponding columns $\vec{h}_{i}, \vec{h}_{j}, \vec{h}_{k}$ are linearly dependent. We denote by $l_{x y}$ the line passing through the points $x$ and $y$, and we denote by $P_{x y z}$ the plane spanned by three non-collinear points $x, y, z$. Let $\vec{x}=\left(x_{1}, x_{2}, \ldots, x_{n}\right) \in \mathbb{F}_{q}^{n}$. Then, the support of the vector $\vec{x}$ is the set $\operatorname{supp}(\vec{x})=\left\{i: x_{i} \neq 0\right\}$. A triple belongs to the line if the support of this triple belongs to the line. The triples intersect at the point $i$ if their supports intersect at the point $i$.

\section{Properties of $i$-components}

Next, we present theorems describing the properties of the $i$-components of code $\mathbb{H}$. Let the subcode $\mathbb{H}_{l}$ of code $\mathbb{H}$ be defined by the line $l$. We consider the pencil of lines $l_{1}, l_{2}, \ldots, l_{(n-1) / q}$ through a point $i$. It is known [1] that

$$
R_{i}=\mathbb{H}_{l_{1}}+\mathbb{H}_{l_{2}}+\cdots+\mathbb{H}_{l_{(n-1) / q}}
$$

Theorem 1. Let a vector $\vec{u}=\left(u_{1}, u_{2}, \ldots, u_{n}\right) \in R_{i}$ and a component $u_{x}$ of the vector $\vec{u}$ be nonzero, $x \neq i$. Then on the line $l_{i x}$ there exists a point $y$ distinct from the points $i, x$ and such that component $u_{y}$ of the vector $\vec{u}$ is nonzero.

P r oof. The basis of the subspace $R_{i}$ is formed by all linearly independent triples of the code $\mathbb{H}$ having 1 in the $i$-th coordinate. Consider representation of the vector $\vec{u}$ with respect to the basis. From the conditions of the theorem, it follows that in this representation is a triple whose support contains points $i, x$ and a point which is on the line $l_{i x}$ and is distinct from the points $i, x$. From formula 1, it follows that the basis triples belonging to the line $l_{i x}$ form a subspace $\mathbb{H}_{l_{i x}}$. The basis triples, that belong to other lines from the pencil of lines containing the point $i$, intersect with the basis triples, that lie on the line $l_{i x}$, only at one point $i$. The theorem is proved.

Theorem 2. Let $i \neq j$, a vector $\vec{u}=\left(u_{1}, u_{2}, \ldots, u_{n}\right) \in R_{i}+R_{j}$, a component $u_{x}$ of the vector $\vec{u}$ be nonzero and the point $x$ does not lie on $l_{i j}$. Then on the plane $P_{i j x}$ there exists a point $y$ distinct from the points $i, j, x$ and such that component $u_{y}$ of the vector $\vec{u}$ is nonzero.

Proof. This theorem is proved similarly to the previous one. The basis triples of $R_{i}+R_{j}$ that lie on the plane $P_{i j x}$ form a subspace. The lines from the pencil of lines containing the point $i$ either lie on the plane $P_{i j x}$ or intersect with this plane at only one point $i$. The lines of the pencil of lines through the point $j$ have the same property. The theorem is proved. 


\section{Example 1}

Next, we describe the constructions of admissible families of components of code $\mathbb{H}$.

Example 1 .

In the parity-check matrix $H$ of the Hamming code $\mathbb{H}$ of length $n=\left(q^{m}-1\right) /(q-1)$, we choose $m$ linearly independent columns. We assume that we have chosen the columns $\vec{h}_{1}, \vec{h}_{2}, \ldots, \vec{h}_{m}$. Let $(\Lambda \cup\{\overrightarrow{0}\}) \subset \mathbb{F}_{q}^{m}$ be a code containing $t$ nonzero vectors $\vec{\lambda}_{1}, \vec{\lambda}_{2}, \ldots, \vec{\lambda}_{t}$, the weight of each of them be greater than or equal to three. Let the distance between any two distinct vectors from the set $\Lambda=\left\{\vec{\lambda}_{1}, \vec{\lambda}_{2}, \ldots, \vec{\lambda}_{t}\right\}$ be greater than or equal to five. With each vector $\vec{\lambda}_{s}=\left(\lambda_{s 1}, \lambda_{s 2}, \ldots, \lambda_{s m}\right)$ of length $m$ we associate a vector $\vec{u}_{s}$ of length $n$, where $s \in\{1, \ldots, t\}$. Let

$$
\mu_{s} \vec{h}_{i_{s}}=\lambda_{s 1} \vec{h}_{1}+\lambda_{s 2} \vec{h}_{2}+\cdots+\lambda_{s m} \vec{h}_{m}
$$

where $\mu_{s} \in \mathbb{F}_{q}, i_{s} \in\{1,2, \ldots, n\}$. Then we put

$$
\vec{u}_{s}=\left(\lambda_{s 1}, \lambda_{s 2}, \ldots, \lambda_{s m}, 0, \ldots, 0,-\mu_{s}, 0, \ldots, 0\right) .
$$

The support of the vector $\vec{u}_{s}$ belongs to $\{1,2, \ldots, m\} \cup\left\{i_{s}\right\}$. Since the Hamming code $\mathbb{H}$ forms a null space of parity check matrix $H$, we have $\vec{u}_{s} \in \mathbb{H}$. Thus, based on vectors of length $m$ from the set $\Lambda$, we constructed a family of components $R_{i_{1}}+\vec{u}_{1}, R_{i_{2}}+$ $\vec{u}_{2}, \ldots, R_{i_{t}}+\vec{u}_{t}$ of the $q$-ary Hamming code $\mathbb{H}$ of length $n=\left(q^{m}-1\right) /(q-1)$.

Etzion and Vardy [3] used a set of linearly independent columns of the parity-check matrix of the Hamming code for constructing the full-rank binary 1-perfect codes.

Next, we show that the family of components from Example 1 is admissible.

Proposition 1.

Let $s \in\{1,2, \ldots, t\}$. Then, $\vec{u}_{s} \notin R_{i_{s}}$.

Proof. From the construction it follows that the support of the vector $\vec{u}_{s}=$ $\left(u_{1}, u_{2}, \ldots, u_{n}\right)$ belongs to $\{1,2, \ldots, m\} \cup\left\{i_{s}\right\}$ and column $\vec{h}_{i_{s}}$ is a linear combination of three or more columns from the set $\left\{\vec{h}_{1}, \vec{h}_{2}, \ldots, \vec{h}_{m}\right\}$. Since the columns $\vec{h}_{1}, \vec{h}_{2}, \ldots, \vec{h}_{m}$ are linearly independent, it follows that for $x \in\{1,2, \ldots, m\}$ no linear combination of columns $\vec{h}_{i_{s}}$ and $\vec{h}_{x}$ does not belong to $\left\{\vec{h}_{1}, \vec{h}_{2}, \ldots, \vec{h}_{m}\right\} \backslash\left\{\vec{h}_{x}\right\}$. Thus from Theorem 1 we have that $\vec{u}_{s} \notin R_{i_{s}}$. The proposition is proved.

Theorem 3 .

The family of the component $R_{i_{1}}+\vec{u}_{1}, R_{i_{2}}+\vec{u}_{2}, \ldots, R_{i_{t}}+\vec{u}_{t}$ of the q-ary Hamming code $\mathbb{H}$ of length $n$ is admissible.

P r o o f. Let $r, s \in\{1,2, \ldots, t\}, r \neq s$. Then, we show that

$$
\left(R_{i_{r}}+\vec{u}_{r}\right) \cap\left(R_{i_{s}}+\vec{u}_{s}\right)=\varnothing .
$$

In order to satisfy the equality 2 , it suffices to show that $\vec{u}_{r}-\vec{u}_{s} \notin R_{i_{r}}+R_{i_{s}}$. We consider several cases. 
1. Let $i_{r}=i_{s}$.

Then, the vectors $\vec{u}_{r}$ and $\vec{u}_{s}$ are linearly dependent. From the construction of vectors $\vec{u}_{r}$ and $\vec{u}_{s}$ implies that the weight of vector $\vec{u}_{r}-\vec{u}_{s}$ is greater than or equal to six. Hence, arguing as in the proof of Proposition 1, we obtain that $\vec{u}_{r}-\vec{u}_{s} \notin R_{i_{r}}$.

2. Let $i_{r} \neq i_{s}$.

Then, we show that $\vec{u}_{r}-\vec{u}_{s} \notin R_{i_{r}}+R_{i_{s}}$. By Theorem 2, it suffices to show that the support of vector $\vec{u}_{r}-\vec{u}_{s}$ contains a point $x$ not lying on the line $l_{i_{r} i_{s}}$ and such that no other point (distinct from the points $i_{r}, i_{s}, x$ ) of the support does not belong to the plane $P_{i_{r} i_{s} x}$.

2.1. Let the columns $\vec{h}_{i_{r}}$ and $\vec{h}_{i_{s}}$ be such that as a result of any linear combination of these columns, one obtains a column, which can be represented linear combination of three or more columns from the set $\left\{\vec{h}_{1}, \vec{h}_{2}, \ldots, \vec{h}_{m}\right\}$.

The support of vector $\vec{u}_{r}-\vec{u}_{s}$ belongs to $\{1,2, \ldots, m\} \cup\left\{i_{r}\right\} \cup\left\{i_{s}\right\}$. Consequently, the point $x$ belong to $\{1,2, \ldots, m\}$. Since the columns $\vec{h}_{1}, \vec{h}_{2}, \ldots, \vec{h}_{m}$ are linearly independent, it is obvious that none of the columns of the set $\left\{\vec{h}_{1}, \vec{h}_{2}, \ldots, \vec{h}_{m}\right\} \backslash\left\{\vec{h}_{x}\right\}$ is not a linear combination of the columns $\vec{h}_{i_{r}}, \vec{h}_{i_{s}}, \vec{h}_{x}$. Consequently, $\vec{u}_{r}-\vec{u}_{s} \notin R_{i_{r}}+R_{i_{s}}$.

2.2. Let the columns $\vec{h}_{i_{r}}$ and $\vec{h}_{i_{s}}$ be such that as a result of linear combination of these columns, one obtains the column $\vec{h}$ which can be represented linear combination of two columns $\vec{h}_{y^{\prime}}, \vec{h}_{y^{\prime \prime}}$ from the set $\left\{\vec{h}_{1}, \vec{h}_{2}, \ldots, \vec{h}_{m}\right\}$.

2.2.1. If at least one of the points $y^{\prime}, y^{\prime \prime}$ do not belong to the support of vector $\vec{u}_{r}-\vec{u}_{s}$, then $\vec{u}_{r}-\vec{u}_{s} \notin R_{i_{r}}+R_{i_{s}}$.

2.2.2. Let $y^{\prime}, y^{\prime \prime}$ be the points belonging to the support of vector $\vec{u}_{r}-\vec{u}_{s}$. Then, we choose a point in the support of the vector $\vec{u}_{r}-\vec{u}_{s}$ which is distinct from the points $i_{r}, i_{s}, y^{\prime}, y^{\prime \prime}$. Such a choice is possible by the construction of vectors $\vec{u}_{r}, \vec{u}_{s}$. The support of the vector $\vec{u}_{r}-\vec{u}_{s}$ belongs to $\{1,2, \ldots, m\} \cup\left\{i_{r}\right\} \cup\left\{i_{s}\right\}$. Hence, the points $x, y^{\prime}, y^{\prime \prime}$ belong to $\{1,2, \ldots, m\}$ and are not collinear. Consider any other linear combination of columns $\vec{h}_{i_{r}}, \vec{h}_{i_{s}}$ as a result of which, we obtain a column that is linearly independent from the column $h$. Since the distance between the vectors $\vec{\lambda}_{r}$ and $\vec{\lambda}_{s}$ greater than or equal to five and columns $\vec{h}_{1}, \vec{h}_{2}, \ldots, \vec{h}_{m}$ are linearly independent, it follows that the distance between the columns $\vec{h}_{r}$ and $\vec{h}_{s}$ is also greater than or equal to five. Consequently, as a result of the linear combination, we obtain a column which is a linear combination of three or more columns from the set $\left\{\vec{h}_{1}, \vec{h}_{2}, \ldots, \vec{h}_{m}\right\}$. Thus, $\vec{u}_{r}-\vec{u}_{s} \notin R_{i_{r}}+R_{i_{s}}$.

2.3. Let the columns $\vec{h}_{i_{r}}$ and $\vec{h}_{i_{s}}$ be such that as a result of a linear combination of these columns, we obtain the column from the set $\left\{\vec{h}_{1}, \vec{h}_{2}, \ldots, \vec{h}_{m}\right\}$. Then the same arguments as in the previous case, we obtain that $\vec{u}_{r}-\vec{u}_{s} \notin R_{i_{r}}+R_{i_{s}}$. The theorem is proved. 


\section{$4 \quad$ Examples 2 and 3}

Next, we give Examples 2 and 3. The family of components in these examples are constructed in exactly the same way as in Example 1.

\section{Example 2.}

Let $(\Lambda \cup\{\overrightarrow{0}\}) \subset \mathbb{F}_{3}^{m}$ bet a code containing $t$ nonzero vectors $\vec{\lambda}_{1}, \vec{\lambda}_{2} \ldots, \vec{\lambda}_{t}$, the weight of each of them be greater than or equal to three. Let also the distance between any two distinct vectors in $\Lambda=\left\{\vec{\lambda}_{1}, \vec{\lambda}_{2} \ldots, \vec{\lambda}_{t}\right\}$ be greater than or equal to three. Then, the set $\Lambda$ corresponds to admissible family of component of the ternary Hamming code of length $n=\left(3^{m}-1\right) / 2$.

Example 3 .

In the parity-check matrix $H$ of the binary Hamming code $\mathbb{H}$ of length $n=2^{m}-1$, we choose $m$ linearly independent columns. We assume that we have chosen the columns $\vec{h}_{1}, \vec{h}_{2}, \ldots, \vec{h}_{m}$. In the parity-check matrix $H$, we also choose $k$ columns which are linear combination of two columns from $\left\{\vec{h}_{1}, \vec{h}_{2}, \ldots, \vec{h}_{m}\right\}$. We assume that these columns are the columns $\vec{h}_{m+1}, \vec{h}_{m+2}, \ldots, \vec{h}_{m+k}$. Let $(\Lambda \cup\{\overrightarrow{0}\}) \subset \mathbb{F}_{2}^{m+k}$ be a code contains $t$ nonzero vectors $\vec{\lambda}_{1}, \vec{\lambda}_{2}, \ldots, \vec{\lambda}_{t}$, the weight of each of them be greater than or equal to $3 k+3$. Let the distance between any two distinct vectors in $\Lambda=\left\{\vec{\lambda}_{1}, \vec{\lambda}_{2} \ldots, \vec{\lambda}_{t}\right\}$ be greater than or equal to $3 k+3$. With eeach vector $\vec{\lambda}_{s}=\left(\lambda_{s 1}, \lambda_{s 2}, \ldots, \lambda_{s m+k}\right)$ of length $m+k$ same way as in Example 1, we associate a vector $\vec{u}_{s}$ of length $n=2^{m}-1$, where $s \in\{1, \ldots, t\}$. Then, the set $\Lambda$ corresponds to admissible family of components of the binary Hamming code of length $n=2^{m}-1$.

The proof of the fact that the families of components in Examples 2 and 3 are admissible is similar to the proof of Theorem 3. In the case of ternary codes, we should take in account the features of the Galois field $\mathbb{F}_{3}$. Let $\vec{x}, \vec{y} \in \mathbb{F}_{3}^{m}$. Then, it is obvious that if $d(\vec{x}, \vec{y})=m$ and vectors $\vec{x}, \vec{y}$ does not contain zero components, then they are linearly dependent.

\section{$5 \quad$ Embedding in perfect code}

Next, we prove a theorem on the embeddability.

By $\vec{e}_{i}$ we denote a vector of length $n$, where $i$-th component is equal to 1 and other components are equal to 0 .

Let

$$
\mathbb{T}=\left(\mathbb{H} \backslash \bigcup_{s=1}^{t}\left(R_{i_{s}}+\vec{u}_{s}\right)\right) \cup\left(\bigcup_{s=1}^{t}\left(R_{i_{s}}+\vec{u}_{s}+\mu_{s} \cdot \vec{e}_{i_{s}}\right)\right)
$$

By Theorem 3 , the family of component $R_{i_{1}}+\vec{u}_{1}, R_{i_{2}}+\vec{u}_{2}, \ldots, R_{i_{t}}+\vec{u}_{t}$ of $q$-ary Hamming code $\mathbb{H}$ is admissible (similar theorems on the admissibility of the family component are 
valid for the codes from Examples 2 and 3 ). Consequently, the set $\mathbb{T}$ is $q$-ary 1-perfect code of length $n$, see 3 , 4. By Proposition 1, the code $\mathbb{T}$ contains the zero vector.

\section{Theorem 4.}

Every $q$-ary code of length $m$ and minimum distance 5 (for $q=3$ the minimum distance is 3) can be embedded in a q-ary 1-perfect code of length $n=\left(q^{m}-1\right) /(q-1)$. Every binary code of length $m+k$ and minimum distance $3 k+3$ can be embedded in a binary 1-perfect code of length $n=2^{m}-1$.

P r o of. From the construction of the admissible family of component of $q$-ary code $\mathbb{H}$ in Example 1 and formula 3 , it follows that every $q$-ary code $\Lambda \cup\{\overrightarrow{0}\}$ of length $m$ and minimum distance 5 can be embedded (in the strong sense) in the $q$-ary 1 -perfect code $\mathbb{T}$ of length $n=\left(q^{m}-1\right) /(q-1)$.

From the construction of the admissible family of component of ternary code $\mathbb{H}$ in Example 2 and Formula [3, it follows that every ternary code $\Lambda \cup\{\overrightarrow{0}\}$ of length $m$ and minimum distance 3 can be embedded (in the strong sense) in a ternary 1-perfect code $\mathbb{T}$ of length $n=\left(3^{m}-1\right) / 2$.

From the construction of admissible family of component of binary code $\mathbb{H}$ in Example 3 and Formula 3, it follows that every binary code $\Lambda \cup\{\overrightarrow{0}\}$ of length $m+k$ and minimum distance $3 k+3$ can be embedded in a binary 1 -perfect code $\mathbb{T}$ of length $n=2^{m}-1, k \geq 0$. In the case of binary codes of Example 3 the embedding is not strong. The theorem is proved.

\section{References}

[1] Romanov A. M. On partitions of $q$-ary Hamming codes into disjoint components. // Discrete Analysis and Operations Research. - 2004. Ser. 1, - V. 11, N 3. - P. 80-87.

[2] Avgustinovich S. V., Krotov D.S. Embedding in a perfect code // Journal of Combinatorial Designs. - 2009. - V. 17, N 5. - P. 419-423.

[3] Etzion T., Vardy A. Perfect binary codes: Constructions, properties and enumeration // IEEE Trans. Inf. Theory. - 1994. - V. 40, N 3. - P. 754-763.

[4] Phelps K. T., Villanueva M. Ranks of $q$-ary 1-perfect codes // Designs, Codes and Cryptogr. - 2002. — V. 27, N 1-2. — P. 139-144.

[5] Romanov A. M. Survey of Methods for Construction of Nonlinear Perfect Binary Codes // Journal of Applied and Industrial Mathematics. - 2008. - V. 2, N 2. P. $252-269$. 